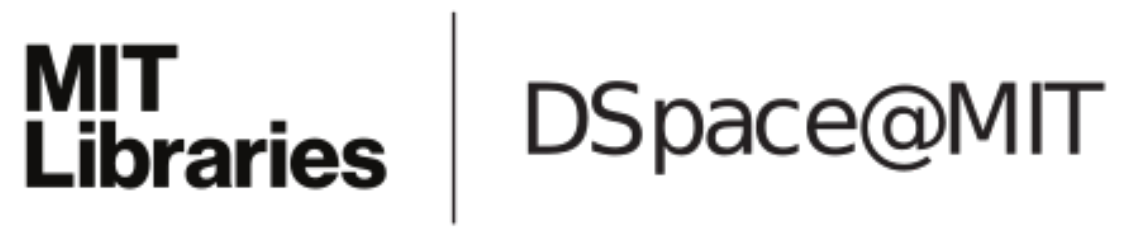

\author{
MIT Open Access Articles
}

Ribocomputing devices for sophisticated in vivo logic computation

The MIT Faculty has made this article openly available. Please share how this access benefits you. Your story matters.

Citation: Green, Alexander A.; Kim, Jongmin; Ma, Duo; Silver, Pamela A.; Collins, James J. and Yin, Peng. "Ribocomputing Devices for Sophisticated in Vivo Logic Computation." NANOCOM'16, Proceedings of the 3rd ACM International Conference on Nanoscale Computing and Communication, September 28-30 2016, New York, New York, Association for Computing Machinery (ACM), September 2016

As Published: http://dx.doi.org/10.1145/2967446.2970373

Publisher: Association for Computing Machinery (ACM)

Persistent URL: http://hdl.handle.net/1721.1/109202

Version: Author's final manuscript: final author's manuscript post peer review, without publisher's formatting or copy editing

Terms of use: Creative Commons Attribution-Noncommercial-Share Alike 


\section{Ribocomputing devices for sophisticated in vivo logic computation}

\author{
Alexander A. Green \\ Biodesign Center for Molecular \\ Design and Biomimetics \\ Arizona State University \\ Tempe, AZ 85287 \\ alexgreen@asu.edu \\ Pamela A. Silver \\ Department of Systems Biology \\ Harvard Medical School \\ Boston, MA 02115 \\ pamela_silver@hms.harvard.e \\ du \\ Jongmin Kim \\ Wyss Institute for Biologically \\ Inspired Engineering \\ Harvard University \\ Boston, MA 02115 \\ jongmin.kim@wyss.harvard.edu \\ James J. Collins \\ Institute for Medical Engineering and \\ Science, Massachusetts Institute of \\ Technology \\ Cambridge, MA 02139 \\ jimjc@mit.edu
}

\author{
Duo Ma \\ Biodesign Center for Molecular \\ Design and Biomimetics \\ Arizona State University \\ Tempe, AZ 85287 \\ duo.ma@asu.edu \\ Peng Yin \\ Wyss Institute for Biologically \\ Inspired Engineering \\ Harvard University \\ Boston, MA 02115 \\ peng_yin@hms.harvard.edu
}

\begin{abstract}
Synthetic biology aims to create functional devices, systems, and organisms with novel and useful functions taking advantage of engineering principles applied to biology. Despite great progress over the last decade, an underlying problem in synthetic biology remains the limited number of high-performance, modular, composable parts. A potential route to solve parts bottleneck problem in synthetic biology utilizes the programmability of nucleic acids inspired by molecular programming approaches that have demonstrated complex biomolecular circuits evaluating logic expressions in test tubes. Using a library of de-novo-designed toehold switches with orthogonality and modular composability, we demonstrate how toehold switches can be incorporated into decision-making RNA networks termed ribocomputing devices to rapidly evaluate complex logic in living cells. We have successfully demonstrated a 4-input AND gate, a 6-input OR gate, and a 12-input expression in disjunctive normal form in E. coli. The compact encoding of ribocomputing system using a library of modular parts is amenable to aggressive scale-up towards complex control of in vivo circuitry towards autonomous behaviors and biomedical applications.
\end{abstract}

\section{CCS Concepts}

- Applied computing Biological networks

Permission to make digital or hard copies of all or part of this work for personal or classroom use is granted without fee provided that copies are not made or distributed for profit or commercial advantage and that copies bear this notice and the full citation on the first page. Copyrights for components of this work owned by others than the author(s) must be honored. Abstracting with credit is permitted. To copy otherwise, or republish, to post on servers or to redistribute to lists, requires prior specific permission and/or a fee. Request permissions from Permissions@acm.org.

NANOCOM'16, September 28 - 30, 2016, New York, NY, USA

Copyright is held by the owner/author(s). Publication rights licensed to ACM.

ACM 978-1-4503-4061-8/16/09 ..\$15.00

DOI: http://dx.doi.org/10.1145/2967446.2970373

\section{Keywords}

synthetic biology, toehold switch, RNA network.

\section{INTRODUCTION}

Synthetic biology aims to address challenging problems in the fields of biotechnology and medicine by constructing novel and complex circuits by applying engineering principles. Starting from the first demonstration of synthetic circuits in bacteria more than a decade ago, milestone achievements have demonstrated bistability [4], oscillation [3], feedback [2], and logic processing [7] in living cells. The synthetic biological circuits to date have relied heavily on protein-based regulators that are difficult to scale up due to the limited number of designable parts and significant resource requirements to encode and operate such circuits. More recently, insulation strategies and advanced computer programs were used to assemble 55 sequence elements and 4 computational layers to construct a 3-input consensus circuit [8] -- this circuit required using the equivalent of seven inverter or 2-input logic elements.

A potential route to solve parts bottleneck problem in synthetic biology utilizes the programmability and large sequence space of nucleic-acid-based parts libraries. The advanced understanding of nucleic acid thermodynamics and kinetics allows purely in silico design of novel parts with predictable interaction with existing circuit elements. Thus, we are presented with a great opportunity to exploit the versatile nucleic-acid-based computation in synthetic biology circuits [1,5]. These design strategies can be used in combination with existing protein-based layered circuit architecture for even more complex information processing in cells.

\section{RESULTS}

Here, we report an integrated molecular computation strategy that employs RNA-only circuits to evaluate complex logic in living cells. Central to our current work is a novel class of in vivo RNAbased devices called toehold switches that activate gene expression in bacteria only when they detect a cognate trigger RNA [5] (Figure 1A). The toehold switches take trigger RNAs without any sequence constraints overcoming the limitations of conventional synthetic riboregulators [6]. Further, these toehold 
switches can be forward-engineered to modulate gene expression by several hundred-fold rivaling the best protein-based regulators and provide extremely low levels of crosstalk across large library of components.

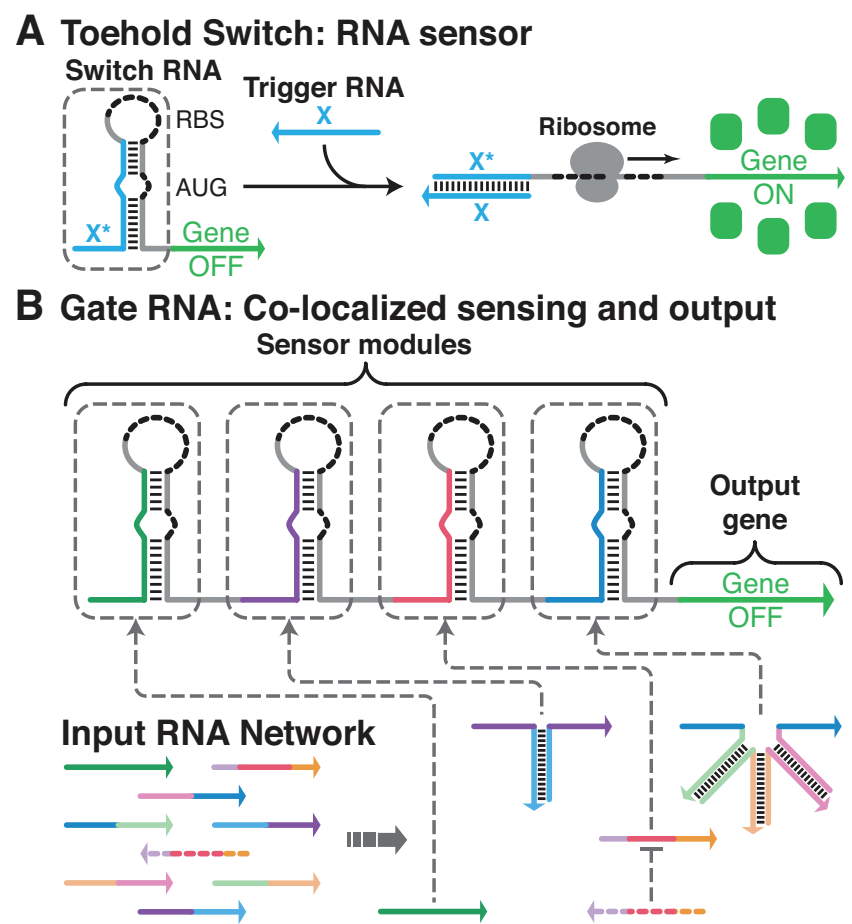

Figure 1. (A) The toehold switches accept trigger RNAs without any sequence constraints to control the translation of downstream gene. (B) Ribocomputing devices consist of colocalized sensor elements utilizing toehold switches and an output gene in an extended gate RNA transcript. This gate RNA can detect the states of input RNA networks.

Using a library of orthogonal devices with modular composability, we demonstrate how toehold switches can be incorporated into decision-making RNA networks termed ribocomputing devices to rapidly evaluate complex logic in living cells (Figure 1B). Each of the concatenated toehold switch sensor contains its own ribosome binding sequence, thereby allowing translation of the downstream gene when any one of the cognate triggers is provided. These concatenated switch hairpins perform the equivalent of OR logic. To integrate AND logic, the trigger sequences were divided into separate input RNA molecules with programmed hybridization domains required to colocalize the split trigger parts. By combining these integrated computation capabilities, we have successfully demonstrated a 4-input AND gate, a 6-input OR gate, and a 12-input expression in disjunctive normal form in E. coli, surpassing the complexity of previous works.

\section{CONCLUSIONS}

We have shown that the integrated molecular computation strategy using ribocomputing devices allow cellular computation of complex Boolean logic networks. The ribocomputing devices utilize the programmable sequence recognition property of nucleic acids, thereby greatly expanding the library of orthogonal, highperformance parts in synthetic biology. Further, ribocomputing devices colocalize several sensing elements and output gene in a single extended transcript thereby reducing the diffusive signal losses and improving signal transduction. These design strategies can be used in combination with existing protein-based layered circuit architecture for even more complex information processing in cells. The generality of ribocomputing devices points towards a possibility of minimal platform and context dependence [9]. This suggests that the ribocomputing devices may be easily adapted to other bacterial hosts for metabolic engineering and biofuel production and to eukaryotic hosts with appropriate design changes.

\section{ACKNOWLEDGMENTS}

We thank K. Pardee, X. Chen, and A. Chavez for helpful discussions. This work was supported by a DARPA Living Foundries grant (HR001112C0061) to P.A.S., P.Y., and J.J.C.; NSF ERA SynBio grant (1540214) and Wyss Institute funds to P.Y.; an ONR MURI Program to J.J.C.; and startup funds provided by Arizona State University to A.A.G. J.K. acknowledges the support of Wyss Institute Director's CrossPlatform Fellowship.

\section{REFERENCES}

[1] Chappell, J., Takahashi, M.K., and Lucks, J.B. (2015). Creating small transcription activating RNAs. Nature Chemical Biology 11, 214-220.

[2] Daniel, R., Rubens, J.R., Sarpeshkar, R., and Lu, T.K. (2013). Synthetic analog computation in living cells. Nature 497, 619-623.

[3] Elowitz, M.B., and Leibler, S. (2000). A synthetic oscillatory network of transcriptional regulators. Nature 403, 335-338.

[4] Gardner, T.S., Cantor, C.R., and Collins, J.J. (2000). Construction of a genetic toggle switch in Escherichia coli. Nature 403, 339-342.

[5] Green, A.A., Silver, P.A., Collins, J.J., and Yin, P. (2014). Toehold Switches: De-Novo-Designed Regulators of Gene Expression. Cell 159, 925-939.

[6] Isaacs, F.J., Dwyer, D.J., Ding, C.M., Pervouchine, D.D., Cantor, C.R., and Collins, J.J. (2004). Engineered riboregulators enable post-transcriptional control of gene expression. Nature Biotechnology 22, 841-847.

[7] Moon, T.S., Lou, C., Tamsir, A., Stanton, B.C., and Voigt, C.A. (2012). Genetic programs constructed from layered logic gates in single cells. Nature 491, 249-253.

[8] Nielsen, A.A.K., Der, B.S., Shin, J., Vaidyanathan, P., Paralanov, V., Strychalski, E.A., Ross, D., Densmore, D., and Voigt, C.A. (2016). Genetic circuit design automation. Science 352.

[9] Pardee, K., Green, A.A., Ferrante, T., Cameron, D.E., DaleyKeyser, A., Yin, P., and Collins, J.J. (2014). PaperBased Synthetic Gene Networks. Cell 159, 940-954. 